\title{
Marcadores reduzidos para a avaliação da personalidade em adolescentes
}

\author{
Neson Haudk Filho- UnivesidadeFedeal doRioGrandedbSul, PatoAlegye RioGrandedbSul, Brasil \\ MarcoAntônioPereira Tèxeira - UnivesidadeFederal dbRioGrandedoSul, ProtoAlegęe, RioGrandedbSul, Brasil \\ Wager deLara Madhadb- UnivesidadeFeedaral dbRioGrandedoSul, PartoAlege, RioGrandedoSul, Brasil \\ DeriseRuschd Bandira - UnivesidadeFedeal doRioGrandedbSul, PatoAlege, RioGrandedoSul, Brasil
}

\begin{abstract}
Resumo
Os Marcadores Reduzidos para a Avaliação da Personalidade mensuram os Cinco Grandes Fatores (CGF) mediante 25 adjetivos comumente utilizados para descrever diferenças individuais em português brasileiro. Contudo, até o momento, suas propriedades psicométricas com adolescentes não haviam sido investigadas. Dessa maneira, o presente estudo teve como objetivo avaliar a estrutura fatorial do instrumento em duas amostras de estudantes adolescentes. 0 primeiro estudo contou com 208 estudantes escolares (média de idades=15,97; DP=1,00). Análises de componentes principais do instrumento revelaram uma estrutura de cinco componentes oblíquos com apenas 20 marcadores. Esse modelo foi testado em uma segunda amostra com 280 estudantes (média de idades=15,53; DP=1,00) de forma confirmatória contra modelos alternativos. Os índices de ajuste também favoreceram um modelo de cinco fatores oblíquos. Os resultados, em conjunto, sugerem a adequação de uma versão do instrumento com apenas 20 marcadores para uso com adolescentes. Palamasdhave Cinco Grandes Fatores; Marcadores da personalidade; Adolescentes; Instrumento de avaliação; Análise Fatorial Confirmatória.
\end{abstract}

\section{Brazilian mini-markers for assessing personality in adolescents}

\begin{abstract}
Brazilian Mini-Markers of Personality measure the Five-Factor Model with 25 adjectives frequently used for describing individual differences in Brazilian Portuguese. However, up to date, there were no studies focusing on its psychometric properties in adolescent groups. Therefore, the aim of the present study was to evaluate the factor structure of the instrument in two adolescent student samples. The Study I used data from 208 high school students (mean age=15.97; $\mathrm{SD}=1.00$ ) and showed an oblique solution composed of five principal components and 20 markers. This structure was then tested with confirmatory factor analysis using data from 280 school and high school students (mean age=15.53; $\mathrm{SD}=1.00$ ). Fit indexes showed that an oblique five-factor model performed better than theoretically-derived concurrent structures. Results showed that a 20 marker version of the instrument is adequate for use with adolescents in Brazil. Keywards Five-Factor Model; Personality markers; Adolescents; Psychometric instrument; Confirmatory Factor Analysis.
\end{abstract}

\section{Mancadores reducidos para la evaluación de la personalidad en adolescentes}

\begin{abstract}
Resumen
Los Marcadores Reducidos para la Evaluación de la Personalidad mensuran los Cinco Grandes Factores (CGF) mediante 25 adjetivos comúnmente utilizados para describir diferencias individuales en portugués brasileño. Todavía, hasta ahora, sus propiedades psicométricas con adolescentes no habían sido investigadas. De esa manera, el presente estudio tuvo como objetivo evaluar la estructura factorial del instrumento en dos muestras de estudiantes adolescentes. El primer estudio contó con 208 estudiantes (promedio de edades=15,97; DP=1,00). Análisis de componentes principales del instrumento revelaron una estructura de cinco componentes oblicuos con apenas 20 marcadores. Ese modelo fue testado en una segunda muestra con 280 estudiantes (promedio de edades=15,53; $\mathrm{DP}=1,00$ ) de forma confirmatoria contra modelos alternativos. Los índices de ajuste también favorecieron un modelo de cinco factores oblicuos. Los resultados, en conjunto, sugieren la adecuación de una versión del instrumento con sólo 20 marcadores para uso con adolescentes.

Palabrasdave Cinco Grandes Factores; Marcadores de personalidad; Adolescentes; Instrumento de evaluación; Análisis Factorial Confirmatoria.
\end{abstract}

O modelo dos Cinco Grandes Fatores (CGF) resume um conjunto complexo de diferenças individuais em cinco traços básicos universais: Extroversão, Socialização, Conscienciosidade, Neuroticismo e Abertura. Esse modelo surgiu com os trabalhos pioneiros de Fiske, Tupes, Cristal e Norman, entre 1949 e 1963 (para uma revisão, ver Digman, 1990). Atualmente, os CGF têm sido avaliados tipicamente por meio de instrumentos de autorrelato, tais como inventários (e.g., Costa \& McCrae, 2007; Nunes, Hutz \& Nunes, 2010) e adjetivos marcadores (Hutz \& cols., 1998; Thompson, 2008). Escores nessas medidas têm sido relacionados a diversas variáveis psicológicas e biológicas, como, por exemplo, a escolha do ambiente ocupacional (Woods \& Hampson, 2010), 0 funcionamento neurobiológico (Cunningham, Arbuckle, Jahn, Mowrer \& Abduljalil, 2010) e a dependência química (Nunes, Nunes, Cunha \& Hutz, 2009).

O modelo dos CGF também tem se mostrado útil na modelagem dos processos maturacionais da personalidade ao longo do desenvolvimento humano (Kandler \& cols., 2010). Todavia, embora o modelo seja aplicável a diversas faixas etárias, isso não significa que um instrumento particular seja adequado tanto para adultos quanto para crianças e adolescentes. $\mathrm{O}$ uso de instrumentos não-específicos para a faixa etária em questão pode, de fato, levar a vieses indesejáveis na 
avaliação (D el Barrio, Carrasco \& Holgado, 2006). Por isso, diversos instrumentos para a avaliação dos CGF têm sido desenvolvidos em versões focadas especificamente em características típicas de crianças e adolescentes (Baker, Victor, Chambers \& Halverson, 2004; Barbaranelli, Caprara, Rabasca \& Pastorelli, 2003; Hendriks, Kuyper, O ffninga \& Van der Werf, 2008).

No Brasil, Hauck, Machado, Teixeira e Bandeira (no prelo) reduziram os 64 marcadores de Hutz e cols. (1998) a um conjunto de 25 marcadores em uma amostra de estudantes universitários. A versão reduzida do instrumento é composta por adjetivos cotidianamente utilizados pelas pessoas para descrever aspectos típicos da personalidade no modelo dos CGF (e.g., "gentil", "responsável", "pessimista"). No entanto, embora esse instrumento tenha tido como propósito inicial a avaliação dos CGF em indivíduos adultos, os marcadores selecionados são de fácil compreensão. De fato, a literatura sinaliza que adolescentes mostraram, em diversos estudos, competências para responder a instrumentos que se utilizam de termos descritores da personalidade (Del Barrio \& cols., 2006). O uso do instrumento de marcadores desenvolvido por Hauck e cols. (no prelo) em adolescentes, assim, é uma possibilidade não devidamente avaliada até 0 momento. Em virtude disso, o objetivo do presente estudo foi investigar a estrutura fatorial do instrumento em indivíduos da faixa etária adolescente.

A pesquisa foi desenvolvida por meio de dois estudos independentes, em uma estratégia de validação cruzada. No primeiro estudo, investigou-se a estrutura do instrumento em uma amostra de estudantes de uma escola pública com análises de componentes principais. No Estudo II, o modelo encontrado no Estudo I foi testado de forma confirmatória em uma amostra de estudantes de uma escola particular contra outros modelos teoricamente plausíveis. A hipótese de pesquisa foi que uma estrutura de cinco fatores oblíquos representaria, parcimoniosamente, as relações entre os dados em ambas as amostras. Essa estrutura foi relatada no estudo original do instrumento com adultos e em outros estudos na literatura com adolescentes (Baker \& cols., 2004; Del Barrio \& cols., 2006; Hauck \& cols., no prelo).

\section{E studo I}

\section{Participantes}

\section{Método}

Participaram do estudo 208 adolescentes estudantes de uma escola pública da cidade de Santa Maria, no Rio Grande do Sul (média de idades=15,97; $\mathrm{DP}=1,00$; idades entre 14 e 19 anos), 60,1\% do sexo feminino. A amostra foi composta por estudantes do Ensino Médio, sendo 33,2\% do primeiro, 54,8\% do segundo e $12 \%$ do terceiro ano.

\section{Instrumento}

Foram utilizados os 25 marcadores desenvolvidos por Hauck e cols. (no prelo), avaliados em escala Liket, $1=$ Discordb tdalmente, $5=$ Concordb tdalmente Exemplos de marcadores são "comunicativa" para extroversão, "gentil" para socialização, "responsável" para conscienciosidade, "pessimista" para neuroticismo e "intelectual" para abertura. O estudo original relatou coeficientes alfa de Cronbach entre 0,61 e 0,83 para as subescalas do instrumento. Outros instrumentos, não pertinentes a esse estudo, também foram aplicados.

\section{Procedimentos}

Todas as coletas foram feitas em sala de aula, após permissão da direção da escola e de assinatura de termos de consentimento informado pelos participantes e por seus responsáveis legais. 0 projeto foi aprovado pelo Comitê de Ética do Instituto de Psicologia da Universidade Federal do Rio Grande do Sul, protocolo no $07 / 019$.

\section{Análisedos dados}

Foram realizadas análises de componentes principais, com rotação diret ddimin tendo como critério de retenção de fatores a análise paralela de autovalores aleatórios (Horn, 1965). O critério da análise paralela permite reter apenas componentes com explicação incremental em relação a autovalores gerados aleatoriamente com dados criados em simulação. Trata-se de uma estratégia que mantém, tipicamente, um número menor de componentes do que 0 critério de Kaiser tradicional (autovalor $\geq 1$ ). Esses componentes resultam ser, geralmente, também mais fáceis de interpretar, coincidindo com dimensões teoricamente mais relevantes. Utilizou-se o saftuare Factor para as análises estatísticas (Lorenzo-Seva \& Ferrando, 2006). As análises foram feitas a partir de 500 subamostras aleatoriamente derivadas do banco de dados original.

\section{Resultados}

Os dados se mostraram adequados para as análises (Kaiser-Meyer-Olkin=0,76 e teste de Bartlett com $\mathrm{p}<0,001)$. As análises de componentes principais revelaram sete componentes com autovalor $>1$, sendo recomendados pela análise paralela apenas cinco componentes, explicando 52,24\% da variância total dos itens. A solução rotacionada com cinco fatores se 
mostrou interpretável, consistente com os CGF. Todavia, observou-se que três itens migraram de suas dimensões. Esses itens foram: "inibida", originalmente do fator extroversão (relação negativa), com carga acima de 0,30 apenas em neuroticismo; "audaciosa", originalmente do fator abertura, com carga acima de 0,30 apenas em neuroticismo; e "suidadosa", originalmente do fator conscienciosidade, com carga acima de 0,30 apenas em socialização.

A construção do instrumento buscou manter um mesmo número de itens em cada dimensão, a fim de que esse aspecto não causasse diferenças na consistência interna das subescalas (Hauck \& cols., no prelo). Contudo, em função da desigualdade entre os componentes causada pela migração de três adjetivos, decidiu-se proceder à exclusão dos mesmos e a uma nova análise exploratória. Além dos três marcadores referidos, mais dois das dimensões socialização ("compreensiva") e neuroticismo ("deprimida") foram excluídos, tendo como critério apresentarem as menores cargas de suas respectivas dimensões.

Novamente, os dados se mostraram adequados para as análises (Kaiser-Meyer-Olkin $=0,74$ e teste de Bartlett com $p<0,001)$. Emergiram seis componentes principais com autovalor $>1$, sendo, contudo, recomendada a retenção de cinco componentes pela análise paralela (57,75\% da variância total dos itens). A solução se mostrou interpretável, com todos os itens carregando nas dimensões esperadas e sem cargas cruzadas acima de 0,30 em outras dimensões.

0 índice loadingsimpliaty(LS; Lorenzo-Seva, 2003) apresentou incremento para um modelo de 20 itens $(0,61)$ com relação ao modelo de 25 itens $(0,55)$. Esse índice varia de zero a um, ocorrendo o valor máximo quando todas as variáveis do modelo apresentam correlação com seu respectivo fator/ componente e correlações com valor zero para os demais fatores/ componentes (Lorenzo-Seva, 2003). Isso indica que a matriz de cargas se mostrou mais "nítida" e coerente com 0 modelo teórico dos CGF para 0 modelo oblíquo com 20 itens. Os resultados da análise de componentes principais são apresentados na Tabela 1. Intercorrelações, médias, desvios padrão e alfas de Cronbach para as subescalas são apresentados na Tabela 2. As subescalas foram computadas através da média escores individuais nos marcadores e invertendo-se os itens negativos "quieta" e "tímida".

Tabela 1. Cargas fatoriais e variância explicada pelos componentes

\begin{tabular}{|c|c|c|c|c|c|}
\hline & $\mathrm{C}$ & $\bar{A}$ & $\mathrm{~S}$ & $\mathrm{~N}$ & $\bar{E}$ \\
\hline Responsável & 0,85 & & & & \\
\hline Esforcada & 0,83 & & & & \\
\hline Organizada & 0,72 & & & & \\
\hline D edicada & 0,71 & & & & \\
\hline Filosófica & & 0,78 & & & \\
\hline Artística & & 0,67 & & & \\
\hline Criativa & & 0,68 & & & \\
\hline Aventureira & & 0,51 & & & \\
\hline Gentil & & & 0,81 & & \\
\hline Bondosa & & & 0,79 & & \\
\hline Simpática & & & 0,76 & & \\
\hline Amável & & & 0,74 & & \\
\hline Ansiosa & & & & 0,71 & \\
\hline Insegura & & & & 0,70 & \\
\hline Pessimista & & & & 0,69 & \\
\hline Aborrecida & & & & 0,57 & \\
\hline Comunicativa & & & & & 0,81 \\
\hline Quieta & & & & & $-0,81$ \\
\hline Tímida & & & & & $-0,70$ \\
\hline D esembraçada & & & & & 0,46 \\
\hline Variância (\%) & 20,14 & 12,55 & 10,55 & 7,83 & 6,67 \\
\hline
\end{tabular}


Tabela 2. Correlações entre as subescalas, médias, desvios padrão e alfas de Cronbach $(\mathrm{N}=208)$

\begin{tabular}{lccccccc}
\hline Subescala & 1 & 2 & 3 & 4 & 5 & M & DP \\
\hline 1. C & $(0,79)$ & $0,17^{*}$ & $0,36 * * *$ & $-0,06$ & $-0,04$ & 3,22 & 0,68 \\
2. A & & $(0,63)$ & $0,31^{* * *}$ & 0,08 & 0,11 & 2,65 & 0,70 \\
3. S & & & $(0,82)$ & 0,02 & 0,09 & 2,81 & 0,62 \\
4. N & & & $(0,59)$ & $-0,21 * *$ & 2,21 & 0,68 \\
5. E & & & & $(0,69)$ & 2,75 & 0,77 \\
\hline
\end{tabular}

Nota: $\mathrm{C}=$ Conscienciosidade; $\mathrm{A}=\mathrm{Abertura;} \mathrm{S}=$ Socialização; $\mathrm{N}=$ =Neuroticismo; $\mathrm{E}=\mathrm{Extroversão.} \mathrm{M}=$ =média; $\mathrm{DP}=$ desvio padrão. Os valores na diagonal representam a consistência interna das subescalas (Alphade Cronbach). *p<0,01; **p<0,01;**p<0,001.

\section{Discussão}

A análise de componentes principais com 25 marcadores mostrou um modelo diverso do estudo original com estudantes universitários para a presente amostra de adolescentes. Entretanto, após a exclusão de cinco marcadores (três com cargas acima de 0,30 apenas em uma dimensão distinta daquela esperada e mais dois para equilibrar o número de marcadores por dimensão), uma nova análise mostrou um modelo consistente com a expectativa teórica. Assim, mantiveram-se cinco fatores explicando $57,75 \%$ da variância total dos 20 marcadores, consistindo 0 modelo em quatro marcadores para cada subescala. Observou-se que, apesar de um número menor de itens, os alfas de Cronbach (entre 0,59 e 0,82) se mostraram similares ou ainda melhores que os do estudo original para três dimensões (socialização, conscienciosidade e abertura). Considerando que se trata de um instrumento com apenas quatro itens e que o alfa de Cronbach é sensível ao tamanho das subescalas, os valores encontrados são tidos como adequados para um instrumento dessa natureza.

Quanto aos padrões de correlação entre as subescalas, observou-se que conscienciosidade, socialização e abertura apresentaram correlações significativas entre si ( $\mathrm{r}$ entre 0,17 e 0,36 ). Por outro lado, extroversão e abertura se mostraram relativamente ortogonais com relação a essas dimensões ( $r$, em módulo, entre 0,02 e 0,11), relacionando-se significativamente apenas entre si $(r=-$ 0,28). Esses mesmos padrões de correlação apareceram em muitos estudos e foram primeiramente relatados em uma meta-análise (Digman, 1997). Digman propôs a existência de dois fatores de segunda ordem, $\alpha$ (desejabilidade social, explicando conscienciosidade, socialização e neuroticismo) e $\beta$ (crescimento pessoal, explicando abertura e extroversão). Esses fatores têm sido replicados em meta-análises recentes, tendo também tem sido especificado um fator geral da personalidade (FGP) de terceira ordem para explicar a covariância entre os fatores $\alpha$ e $\beta$ (Rushton $\&$ Irwing, 2008, 2009; van der Linden, Nijenhuis, \& Bakker, 2010).

Portanto, pode ser interessante investigar a plausibilidade desses modelos complexos em uma amostra com adolescentes, o que foi realizado a seguir. No Estudo II, investigou-se o ajuste do modelo de cinco fatores oblíquos contra modelos concorrentes (ortogonal, hierárquico Alfa-Beta e FGP) em uma amostra independente.

\section{Estudo II}

\section{Partiapantes}

\section{Método}

Participaram do estudo 280 adolescentes estudantes de uma escola particular da cidade de Porto Alegre, no Rio Grande do Sul (média de idades $=15,53$; $\mathrm{DP}=1,00$, idades entre 13 e 19 anos), sendo 52,7\% do sexo feminino. A amostra foi composta por estudantes do Ensino Médio, sendo 35,4\% do primeiro ano, $31,1 \%$ do segundo e $32,5 \%$ do terceiro.

\section{Instrumento}

Foram utilizados os 25 marcadores descritos no Estudo I, embora com escala Liket 1=Discordb tdalmente $7=$ Concordo totalmente Outros instrumentos, não pertinentes a este estudo, também foram aplicados.

\section{Procedimentos}

Todas as coletas foram feitas em sala de aula, após permissão da direção da escola e de assinatura de termos de consentimento informado pelos participantes. $\mathrm{O}$ projeto foi aprovado pelo Comitê de Ética do Instituto de Psicologia da Universidade Federal do Rio Grande do Sul, protocolo no 2010008.

\section{Análisedos dados}

Dados faltantes foram substituídos pela média do participante para a realização das análises fatoriais confirmatórias sem a necessidade de estimar médias e interceptos (Byrne, 2001). Foram selecionados apenas os 20 itens resultantes das análises do Estudo I para as análises fatoriais confirmatórias. Com essas análises, buscou-se testar a plausibilidade da estrutura de cinco fatores oblíquos em uma amostra independente de adolescentes. Foi utilizado o satvare AMOS 18.0, com método de estimação Maximm Likeihood. Para avaliação dos modelos, foram utilizados os seguintes 
índices de ajuste: $\chi^{2} /$ graus de liberdade (sendo adequados modelos com valor $<3$ ), Parsimmiaus Nomed Fit Index (PNFI; sendo o melhor aquele com maior valor), ComparativeFit Index (CFI; sendo recomendados valores acima de 0,95), Rot Mean Square Error of Approximation (RMSEA; sendo recomendados valores abaixo de 0,08) e razão Consistent Akaike Infomation Criteion por parâmetros estimados (CAIC/ parâmetros; para comparação entre os modelos, sendo o melhor aquele com menor valor). As recomendações foram baseadas em Byrne (2001).

Em razão da relativa ortogonalidade entre algumas dimensões, tal como evidenciado no Estudo I, e de padrões de correlação semelhantes àqueles relatados em estudos sobre estruturas hierárquicas, foram testados quatro modelos concorrentes para os dados. Esses modelos foram: 1) cinco fatores ortogonais; 2) cinco fatores oblíquos; 3) cinco fatores hierárquicos, com os fatores $\alpha$ (explicando conscienciosidade, socialização e neuroticismo) e $\beta$ (explicando extroversão e abertura); e 4) cinco fatores hierárquicos com os fatores $\alpha$ e $\beta$ de segunda ordem e um FGP, de terceira ordem, explicando os fatores de segunda ordem.

\section{Resultados}

Os resultados das análises fatoriais confirmatórias são apresentados na Tabela 3. 0 modelo com melhor ajuste foi 0 de cinco fatores oblíquos. Esse modelo apresentou maior parcimônia (i.e., a capacidade de explicar envolvendo menor complexidade), obtendo os menores valores $\chi^{2} / \mathrm{g}$ e CAIC/ parâmetros estimados. Obteve também 0 melhor ajuste incremental (i.e., a capacidade de explicar mais do que um modelo nulo hipotético), com os maiores valores TLI e CFI. Contudo, pôde-se observar que os índices PNFI e RMSEA, que avaliam parcimônia e falta de ajuste, respectivamente, não discriminaram os modelos 1,3 e 4. Dessa forma, um teste adicional de diferença de $\chi^{2}$ foi realizado, a fim de avaliar a significância estatística da diferença de ajuste entre esses modelos. 0 modelo de cinco fatores oblíquos apresentou ajuste significativamente melhor do que 0 modelo com os fatores $\alpha$ e $\beta\left(\Delta \chi^{2}=45,84, g=6, p<0,001\right)$ e do que 0 modelo com o FGP $\left(\Delta \chi^{2}=22,29, \mathrm{~g}=4, \mathrm{p}<0,001\right)$.

Tabela 3. Resultados das análises fatoriais confirmatórias

\begin{tabular}{lcccccc}
\hline Modelos & $\chi^{2} / \mathrm{g}$ & PNFI & TLI & CFI & RMSEA & CAIC/ Par. \\
\hline 1. Cinco fatores oblíquos & 2,34 & 0,65 & 0,82 & 0,85 & 0,07 & 14,12 \\
2. Cinco fatores ortogonais & 2,78 & 0,63 & 0,76 & 0,79 & 0,08 & 18,43 \\
3. Hierárquico $\alpha$ e $\beta$ & 2,53 & 0,65 & 0,79 & 0,82 & 0,07 & 16,18 \\
4. Hierárquico FGP & 2,42 & 0,65 & 0,81 & 0,84 & 0,07 & 15,25 \\
\hline
\end{tabular}

Nota: $\alpha=$ fator Alfa; $\beta=$ fator Beta; FGP=fator geral da personalidade; $\mathrm{g}=$ =graus de liberdade; PNFI=ParsimmiasNomed Fit Index; TLI=TukerLevis Index; CFI=Comparative Fit Index; RMSEA=Rot Mean Square Emor of Approximation CAIC/ Par.=Consistent Akaike Information Criteiơ parâmetros estimados

De uma maneira geral, o ajuste do modelo de cinco fatores oblíquos foi melhor do que 0 dos modelos concorrentes. Todavia, os valores para TLI e CFI se mostraram abaixo do valor recomendado de 0,90. Assim, foi feita uma análise de componentes principais suplementar com rotação Diret Oblimin e critério de retenção da análise paralela para investigar a estrutura dos dados de forma exploratória. Essa análise também apontou para uma solução com cinco componentes oblíquos, os quais explicaram $56,74 \%$ da variância total dos itens. O índice Loading Simpliaty (Lorenzo-Seva, 2003) foi de 0,57, menor para o modelo com a presente amostra em relação à amostra do Estudo I $(0,61)$. Ainda assim, contudo, foi maior do que para 0 modelo de 25 marcadores do Estudo I $(0,55)$. A consistência interna das subescalas foi: extroversão $=0,74$, Socialização $=0,80$, Conscienciosidade $=0,76, \quad$ Neuroticismo $=0,55 \quad$ e Abertura $=0,57$.

\section{Discussão}

O s resultados das análises fatoriais confirmatórias forneceram apoio empírico para o modelo de cinco fatores relacionados, e esse modelo apresentou melhor ajuste parcimonioso e incremental, bem como resíduos significativamente menores do que os demais modelos. As subescalas resultantes para esse modelo apresentaram alfas de Cronbach semelhantes aos do Estudo I, variando entre 0,55 e 0,80. Apesar de relativamente baixos, esses valores podem ser considerados adequados para um instrumento reduzido com apenas quatro itens em cada subescala.

O bservou-se, entretanto, que os valores de TLI e CFI desse modelo, ainda que superiores aos demais modelos concorrentes, ficaram abaixo do ponto de corte recomendado, de 0,95. Entretanto, resultados semelhantes foram relatados em diversos estudos prévios de análise fatorial confirmatória para instrumentos que avaliam os CGF (Cooper, Smillie \& Corr, 2010; García, Aluja \& García, 2004; McCrae \& 
cols., 2008; Ruiz \& Jiménez, 2004). Essa tendência é explicada pelo fato de que a complexidade típica de itens de inventários de personalidade geralmente incorre em cargas fatoriais cruzadas não desprezíveis (Borkenau \& O stendorf, 1990). Dessa forma, uma vez que a análise fatorial confirmatória possui a suposição restritiva de que os itens tenham carga em apenas um fator e cargas nulas nos demais, os índices podem sugerir a rejeição do modelo, ainda que o mesmo represente a perspectiva teórica mais ajustada aos dados. Em consonância com essa hipótese, uma análise de componentes principais suplementar também mostrou uma solução consistente com o modelo dos CGF, sem cargas cruzadas maiores que 0,30 nos 20 itens.

\section{Discussão geral e considerações finais}

O presente estudo teve como objetivo investigar a estrutura fatorial de marcadores para a avaliação da personalidade em duas amostras de adolescentes. Embora 0 instrumento tenha sido originalmente desenvolvido para 0 uso com indivíduos adultos, 0 presente estudo forneceu evidências de validade para 0 uso também com adolescentes. Assim, um modelo de cinco fatores oblíquos se mostrou representativo dos dados em duas amostras independentes, tendo sido replicável por meio de análises exploratórias de componentes principais e análise fatorial confirmatória. A consistência interna das subescalas do instrumento também se mostrou adequada, variando de 0,55 a 0,82, valores dentro da expectativa para subescalas com apenas quatro itens. Outras medidas breves amplamente utilizadas também apresentaram consistência interna abaixo de 0,70, tal como 0,68 a 0,81 em Cooper e cols. (2010) e 0,34 a 0,71 em Ehrhart e cols. (2009).

Vale ressaltar, no entanto, que três adjetivos utilizados originalmente com adultos não se comportaram da maneira esperada nas análises do presente estudo. Especificamente, os marcadores "Inibida", "Audaciosa" e "Cuidadosa" apresentaram altas cargas em dimensões não-esperadas. Em virtude disso, é possível que esses descritores tenham tido uma interpretação diversa daquela do contexto universitário. Assim, por exemplo, ser uma pessoa "audaciosa" pode ter sido entendido como denotando hostilidade, antagonismo ou mesmo promiscuidade sexual, uma vez que o marcador apresentou carga acima de 0,30 em neuroticismo. A falta de clareza quanto ao significado atribuído a esses três marcadores motivou sua exclusão da versão para adolescentes do instrumento. Outros dois itens com baixas cargas também tiveram de ser retirados, para que houvesse uma quantidade homogênea de marcadores para cada subescala.

As características representadas pelos CGF da personalidade têm sido entendidas como adaptações psicológicas mantidas na espécie humana em razão do seu papel funcional no Ambiente de Adaptação Evolutiva (Michalski \& Shackelford, 2010). Ou seja, representam domínios de características que, no passado da espécie humana, ajudaram os indivíduos a solucionarem problemas adaptativos, como na competição por recursos ou por parceiras e parceiros sexuais. Os CGF, além disso, são informações essenciais que os indivíduos dentro de uma organização social buscam saber sobre outros indivíduos, a fim de estabelecer quem está e quem não está disposto a colaborar com a ordem social e a distribuição de recursos (Figueredo \& cols., 2005). Todavia, muitas características herdadas por terem sido úteis aos indivíduos quando na fase adulta não necessariamente estão plenamente presentes em indivíduos mais jovens e vice-versa (Bjorklund \& Blasi, 2005). Isso faz com que o estudo da personalidade em uma perspectiva desenvolvimental seja de relevância para uma compreensão aprofundada dos fatores maturacionais envolvidos com a expressão dos traços de personalidade na espécie humana.

Nesse sentido, embora não tenha sido um propósito do estudo avaliar diferenças nos escores brutos no instrumento em função da idade dos indivíduos, foi possível observar uma diferença entre as médias obtidas para as escalas no presente estudo e no estudo original (Hauck \& cols., no prelo). Pontualmente, os participantes adolescentes apresentaram médias mais baixas em todas as escalas do instrumento do que os participantes da amostra universitária de construção do instrumento. Esse resultado é sugestivo de uma possível maturação dos traços de personalidade dos CGF, tópico que vem ganhando espaço na literatura na área. Nesse sentido, há evidências de que, apesar da estabilidade do modelo dos CGF ao longo do tempo, o passar do tempo faz com que haja uma mudança no quanto os indivíduos, em média, apresentam os padrões comportamentais, afetivos e cognitivos característicos de cada dimensão. Por exemplo, Srivastava, John, Gosling e Potter (2003) mostraram a existência de curvas de crescimento distintas para cada um dos CGF em função da idade dos indivíduos. Os autores encontraram que os fatores socialização e conscienciosidade claramente aumentaram no período de tempo entre os 21 e os 60 anos. Além disso, mulheres apresentaram médias mais altas nesses fatores quando controlada a variável idade. Portanto, à luz de resultados como esse, sugere-se que aspectos maturacionais dos CGF no Brasil sejam estudados a partir do instrumento de marcadores 
proposto. 0 fato de a versão do instrumento para adolescentes ser composta por itens que também constam na versão para adultos faz do instrumento um recurso importante em estudos longitudinais, permitindo a utilização e a comparação dos escores em diferentes momentos em estudos de medidas repetidas.

Uma limitação do presente estudo é que não foi possível investigar a invariância da estrutura fatorial do instrumento e das cargas fatoriais quando considerado o sexo biológico dos participantes. Comparações dessa natureza exigiriam um tamanho amostral maior do que o disponível para o presente estudo, sendo um tópico a ser mais bem investigado futuramente. Além disso, seria de grande utilidade estimar a invariância dos parâmetros de cada item do instrumento em meninos e meninas. Para isso, seria adequado empregar procedimentos da Teoria de Resposta ao Item (TRI), como o funcionamento diferencial baseado no modelo Rating Scalepara itens politômicos, de Andrich (1978). Uma segunda limitação diz respeito à natureza não aleatória das amostras utilizadas para as análises. Novos estudos poderiam buscar maior representatividade do contexto nacional mediante procedimentos mais sistemáticos de amostragem. Aumentar a variabilidade amostral quanto à abrangência dos diversos níveis de traço latente para cada escala também favoreceria análises baseadas em modelos da TRI.

De maneira geral, os dois estudos apresentaram resultados consistentes entre si, revelando uma estrutura teoricamente plausível de cinco fatores oblíquos. $O$ presente trabalho apresentou uma versão dos Marcadores Reduzidos para a Avaliação da Personalidade para uso com adolescentes. 0 instrumento, com 20 itens, possui propriedades psicométricas adequadas para uso em estudos futuros no Brasil.

\section{Referências}

Andrich, D. (1978). A rating formulation for ordered response categories. Psychmerika, 43, 561-573.

Baker, S. R., Victor, J. B., Chambers, A. L., \& Halverson, Jr., C. F. (2004). Adolescent personality: a five-factor model construct validation. Assessment, 11(4), 303-315.

Barbaranelli, C., Caprara, G., Rabasca, A., \& Pastorelli, C. (2003). A questionnaire for measuring the Big Five in late childhood. Personality and Individual Differences 34(4), 645-664

Bjorklund, D. F., \& Blasi, C. H. (2005). Evolutionary developmental psychology. Em D. M. Buss (Ed.), Handbodk of Evdutionary Psychdogy (pp. 851-877). Hoboken, Nova Jersey: Wiley \& Sons, Inc.
Borkenau, P., \& Ostendorf, F. (1990). Comparing exploratory and confirmatory factor analysis: a study on the 5-factor model of personality. PessalityandIndividual Differenes, 11(5), 515-524.

Byrne, B. (2001). Structural equation moddingwithAMOS: basic concepts, applications, and programming Nova Iorque: Psychology Press, Taylor \& Francis Group.

Cooper, A. J., Smillie, L. D., \& Corr, P. J. (2010). A confirmatory factor analysis of the Mini-IPIP fivefactor model personality scale. Pessnality and Indvidual Differences, 48(5), 688-691.

Costa, P. T., Jr., \& McCrae, R. R. (2007). NEO PI-R: Inventáno de pessonalidadeNEO revisadb einventánio de incofatares NEO revisadbNEO-FFI-R [V esão anta]. São Paulo: Vetor.

Cunningham, W. A., Arbuckle, N. L., Jahn, A., Mowrer, S. M., \& Abduljalil, A. M. (2010). Aspects of neuroticism and the amygdala: chronic tuning from motivational styles. Neeropsydhogia, 48(12), 3399-3404.

Del Barrio, V., Carrasco, M. A., \& Holgado, F. P. (2006). Factor structure invariance in the Children's Big Five Questionnaire. EuropeanJaumal of Psychdog்al Assessment, 23(2), 158-167.

Digman, J. M. (1990). Personality structure: emergence of the five-factor model. Annual Reviewof Psychlogy, 41, 417-440.

Digman, J. M. (1997). Higher-order factors of the Big Five. Jaumal of Personality and Social Psydhogy, 73(6), 1246-1256.

Ehrhart, M. G., Ehrhart, K. H., Roesch, S. C., ChungHerrera, B. G., Nadler, K., \& Bradshaw, K. (2009). Testing the latent factor structure and construct validity of the Ten-Item Personality Inventory. PessonalityandIndividual Differences, 47, 900-905.

Figueredo, A. J., Sefcek, J. A., Vasquez, G., Brumbach, B. H., King, J. E., \& Jacobs, W. J. (2005). Evolutionary personality psychology. Em D. M. Buss (Ed.), Handbodk of Evdutionary Psychdogy (pp. 851-877). Hoboken, Nova Jersey: Wiley \& Sons, Inc.

García, O., Aluja, A., \& García, L. F. (2004). Psychometric properties of Goldberg's 50 personality markers for the Big Five model. European Jaumal of Psychdogical Assessment, 20(4), 310-319.

Hauck, N., Machado, W. L., Teixeira, M. A. P., \& Bandeira, D. R. (no prelo). Evidências de validade de marcadores reduzidos para a avaliação da personalidade no modelo dos Cinco Grandes Fatores. Psicdøja: Teria ePesquisa 
Hendriks, A. A., Kuyper, H., Offringa, G. J., \& Van der Werf, M. (2008). Assessing young adolescents' personality with the Five-Factor Personality Inventory. Assessment, 15(3), 304-316.

Horn, J. L. (1965), A rationale and test for the number of factors in factor analysis. Psychmotika, 30, 17985.

Hutz, C. S., Nunes, C. H., Silveira, A. D., Serra, J., Anton, M., \& Wieczorek, L. S. (1998). The development of the big five markers for personality assessment in Brazil. Psiđogia: Rellexão eCńtica, 11, 395-411.

Kandler, C., Bleidorn, W., Riemann, R., Spinath, F. M., Thiel, W., \& Angleitner, A. (2010). Sources of cumulative continuity in personality: a longitudinal multiple-rater twin study. Joumal of Pessonality and Social Psydhdogy, 98(6), 995-1008.

Lorenzo-Seva, U. (2003). A factor simplicity index. Psychomerika, 68(1), 49-60.

Lorenzo-Seva, U., \& Ferrando, P. J. (2006). FACTOR: a computer program to fit the exploratory factor analysis model. Behavioral Resernch Mehoos Instrumets and Computess, 38(1), 88-91.

McCrae, R. R., Yagamata, S., Jang, K. L., Riemann, R., Ando, J., O no, Y., Angleitner, A., \& Spinath, F. M. (2008). Substance and artifact in the higher-order factors of the Big Five. Jaumal of Pescnality and Social Psydhdogy, 95(2), 442-455.

Michalski, R. L., \& Shackelford, T. K. (2010). Evolutionary personality psychology: reconciling human nature and individual differences. Pessonality andIndividual Differenes, 48, 509-516.

Nunes, C. H., Hutz, C. S., \& Nunes, M. F. (2010). Bateria Fatoial dePersonalidade(BFP): manual témica São Paulo: Casa do Psicólogo.

Nunes, C. H., Nunes, M. F., Cunha, T. F., \& Hutz, C. S. (2009). Chemical dependence and personality.
Reista Interamericana de Psicologa/ Interamerican Jaumal of Psychdogy, 43(1), 68-76.

Ruiz, V. M., \& Jiménez, J. A. (2004). Estructura de la personalidad: ortogonalidad vesus oblicuidad. Anales dePsicoloǵa, 20(1), 1-13.

Rushton, J. P., \& Irwing, P. (2008). A General Factor of Personality (GFP) from two meta-analysis of the Big Five: Digman (1997) and Mount, Barrick, Scullen, and Rounds (2005). Persomality and Indvidual Differences, 45, 679-683.

Rushton, J. P., \& Irwing, P. (2009). A General Factor of Personality in 16 sets of the Big Five, the Guildford-Zimmerman Temperament Survey, the California Psychological Inventory, and the Temperament and Character Inventory. Pessonality and Individual Differences, 47, 558-564.

Srivastava, S., John, O. P., Gosling, S. D., \& Potter, J. (2003). D evelopment of personality in early and middle adulthood: set like plaster or persistent change? Jaumal of Personality and Social Psychoogy, 84(5), 1041-1053.

Thompson, E. R. (2008). D evelopment and validation of an International English Big-Five MiniMarkers. Pesanality and Individual Differenes 45, 542-548.

van der Linden, D., Te Nijenhuis, J., \& Bakker, A. B. (2010). The General Factor of Personality: a metaanalysis of big five intercorrelations and a criterion-related validity study. Jaumal of Reserch in Personality, 44(3), 315-327.

Woods, S., \& Hampson, S. E. (2010). Predicting adult occupational environments from gender and childhood personality traits. Jaumal of Applied Psychdogy, 95(6), 1045-1057.

Reedbidbem06/ 09/ 2011

Reformuladbem31/ 05/ 2012 Aprovadoem11/ 06/ 2012 
Sobre os autores:

N elson Hauck Filho é psicólogo (UFSM), mestre e doutorando em Psicologia (UFRGS). Possui interesse nas áreas de Psicometria e Estatística, Personalidade, Comportamento Antissocial, motivos para usar bebidas alcoólicas, e Psicologia Evolucionista.

Marco Antonio Pereira Teixeira é psicológo, mestre e doutor em Psicologia (UFRGS). É professor do Programa de Pós-Graduação em Psicologia-UFRGS. Possui interesse em Psicometria, Aconselhamento de Carreira e Processos de Ajustamento de estudantes ao contexto universitário. Coordena o Núcleo de Apoio ao Estudante da UFRGS (NAE-UFRGS).

Wagner de Lara Machado é psicólogo (ULBRA), mestre e doutorando em Psicologia (UFRG S). Tem interesse nas áreas de Psicometria, Psicologia da Saúde e Psicologia Positiva.

Denise Ruschel Bandeira é psicóloga (PUCRS), mestre e doutora em Psicologia (UFRGS). É professora do Programa de Pós-Graduação em Psicologia-UFRGS. Tem experiência na área de Psicologia, com ênfase em Construção e Validade de Testes, Escalas e Outras Medidas Psicológicas, atuando principalmente nos seguintes temas: tradução e adaptação de instrumentos de avaliação psicológica, desenho da figura humana, crianças e adolescentes, estresse e avaliação de programas sociais.

Contato com os autores:

Universidade Federal do Rio Grande do Sul - Instituto de Psicologia. Rua Ramiro Barcelos, 2600, sala 117 - Bairro Santana. CEP 90035-003 - Porto Alegre, RS - Brasil.

E-mail: hauck_psychology@ hotmail.com 